\title{
Posibilidades para un recital en una ciudad poscomunista. Entropología sonora en el "Tigre de los Tatra".
}

\section{Possibilities for a recital in a post-communist city. Sound entropology in the "Tatra Tiger".}

\author{
TIPO DE TRABAJO: \\ Comunicación Virtual.
}

PALABRAS CLAVE:

Arte sonoro, instalación de cuerdas largas, site-specific, entropía.

KEY WORDS:

Sound art, long string installation, site-specific, entropy.

RESUMEN.

A principios de los años 90, los antiguos países comunistas del centro y este europeo sufrieron el desmoronamiento de la economía centralizada tras la caída del muro de Berlin y comenzaron la transición hacia la liberalización. El fracaso del socialismo internacional contra el capitalismo democrático haría devenir el fin de la guerra fría y asentaría el desarrollo del mundo globalizado. Esta pieza artística se emplazaría en el tiempo y espacio de la antigua República Socialista Eslovaca que a principios de los años 2000 se le denominó "El Tigre de los Tatra", una serie de tres conciertos de carácter site-specific donde se intervienen los restos de las estructuras en estado de abandono de tres espacios de la era socialista en la ciudad de Trenčín que de forma simbólica evocan a los iconos del socialismo, la hoz (la intervención en la estructura de un campo de lúpulo), el martillo (la intervención en una fábrica) y la estrella roja (la intervención de los tendederos de un microdistrito socialista).

Haciendo referencia al trabajo de Robert Smithson se hace uso del término "entropía" el cual en su obra tiene que ver con el concepto de tiempo, con el pasado y el futuro. Este cambio, en el contexto particular de la Checoslovaquia comunista donde se ensalzaban los valores e iconos del folk y del nacionalismo, hacia la cultura y economía globalizada de mercado, ha generado la sociedad del desecho, de espacios postindustriales de carácter monumental cargados de entropía que pueden ser reciclados de forma contínua mediante intervenciones artísticas efímeras. En este caso el reciclaje fugaz de estos espacios de la sociedad de consumo se traduce en intervenciones sonoras sobre los vestigios del pasado que se convierten instantáneamente en "monumentos del futuro".

\section{ABSTRACT.}

In the early 1990s, the former communist countries of central and eastern Europe suffered the collapse of the centralized economy after the fall of the Berlin Wall and the transition towards liberalization began. The failure of international socialism against democratic capitalism would lead to the end of the cold war and to the development of the globalized world. This artistic piece would be located in the time and space of the former Slovak Socialist Republic which in the early 2000 s was called "The Tatra Tiger", a series of three site-specific concerts where the remains of the abandoned structures of three spaces of the socialist era in the city of Trenčín that symbolically evoke the icons of socialism, the sickle (the intervention in the structure of a hop field), the hammer (the intervention in A factory) and the red star (the intervention of the clotheslines of a socialist microdistrict). 
Referring to the work of Robert Smithson we make use of the term "entropy" which in his work has to do with the concept of time, past and future. This change, in the particular context of communist Czechoslovakia where the values and icons of folk and nationalism were praised, towards a globalized market culture and economy, has generated a society of waste, of post-industrial spaces with a monumental character charged with entropy which can be recycled continuously through ephemeral artistic interventions. In this case the fleeting recycling of these spaces of the consumer society is translated into sound interventions on the vestiges of the past which instantly become "monuments of the future".

\section{CONTENIDO.}

Introducción.

Para entender el proceso de globalización en el espacio donde tuvo lugar esta práctica artística hemos de remontarnos al inicio de la separación en dos estados en proceso de federalización, Chequia y Eslovaquia. Fue en una de las reformas llevadas a cabo por el presidente de la Checoslovaquia socialista, ideólogo de la primavera de Praga y de nacionalidad eslovaca, el "socialista de rostro humano" Alexander Dubcek en el año 1968. La única reforma de la primavera de Praga que logró sobrevivir despues de la invasión del país por parte de las fuerzas del pacto de Varsovia que reaccionaron con la acción militar contra la aplicación de estas reformas que tenían que ver con las libertades cívicas y que algunos calificaron como "burguesas" ${ }^{1}$ y se mantuvo durante el posterior proceso de "normalización". La sociedad checa y eslovaca ha de esperar a la caída del régimen comunista durante la revolucion del terciopelo para que se les devolviesen las libertades básicas que ya proclamó Dubcek. Pero la escisión completa de los dos estados federales a estados autónomos tiene lugar en el año 1993 con el llamado "divorcio de terciopelo" que aunque considerado como un proceso que hizo gala de haber tenido poco rigor democrático hoy por hoy es vigente.

El cambio estructural de los países del este y centro de europa hacia la economía de mercado despues del fracaso y desmoronamiento del proyecto socialista y el fin de la guerra fría se considera como un hito histórico en el desarrollo de la globalización y el asentamiento de las bases de la situación del presente ${ }^{2}$. Durante los primeros años de democracia en la Eslovaquia federal y en el segundo estado independiente en su historia tras el divorcio, el país esta sumido en una autarquía y las especulaciones sobre el surgimiento de un "tigre económico", es decir, de una incipiente potencia en un país recién abierto y aun por explorar para inversiones extranjeras, esta todavía por llegar. Hemos de esperar hasta la primera década de los años 2000 cuando esto ocurre. Al igual que su vecina y hermana checa, el país entra en la Unión Europea en el 2004 y el gobierno conservador comienza a implementar un programa de reformas con el fin de liberalizar por completo el mercado e industrializar el país con la inversión de otros países. Nacería así el llamado por los economistas "Tigre de los Tatra" ${ }^{3}$, Eslovaquia se convierte en el país en el que más crece su economía en todo el territorio de la Unión Europea durante los años 2002 a 2007.

Como en todos los países poscomunistas, es decir, todos aquellos regímenes socialistas que sufrieron estas fases de transformación, en este proceso de transición y de instauración de un sistema nuevo se generó una gran cantidad de desecho o de ruina en toda la insfraestructura del país, y afirmamos la totalidad porque de manera íntegral la propiedad la gestionaba el estado, con ello nos referimos a las fábricas, la industria, el campo, maquinaria de producción, el tejido urbanístico, su arquitectura y por lo tanto todo el espacio público en general. Es precisamente en estos espacios donde ha tenido lugar esta serie de documentación de tres intervenciones en restos abandonados del pasado socialista, que pretende a partir de la acción artística convertir las ruinas en monumentos temporales del futuro a través de la idea de no-lugar, entropía y con especial interés el concepto de reciclaje en la obra de Robert Smithson. Tres intervenciones sonoras a modo de conciertos y recitales con los objetos y espacios abandonados haciendo uso de los micrófonos piezo-eléctricos para la ejecución y el registro.

\section{Desarrollo.}

La serie de esta producción artística lleva por título "Posibilidades para un recital en una ciudad poscomunista" tiene un carácter documental sobre las intervenciones que tuvieron lugar durante los meses de Marzo a Junio de 2014 en la ciudad de Trenčín (Eslovaquia) bajo la supervisión del prof. Mgr.art. Anton Čierny, director del "Estudio de Comunicaciones Espaciales" de la Facultad de Arte y Diseño de Bratislava y por tanto avalado y financiado por esta institución.

\footnotetext{
${ }^{1}$ GOTT, R. Cuba. A new story. Yale University Press, 2004. 365 p. ISBN 978-84-460-2432-3.

2 FRASSINETI, C. La globalización vista desde los últimos. Translated by AO García; edited by Sal Terrae. 1st. ed. Cittadela Editrice, 2000. 20 p. ISBN 84293-1412-1.

${ }^{3}$ GRANCAY, M and GRANCAY, N. Foreign Direct Investment in Central and Eastern Europe: Post-crisis. 1st. ed; Budapest: Studies in economic transition 2017. Section 4, Szent-Iványi, pp 77-99.
} 
La serie consta de tres partes y cada una de ellas hace referencia a los símbolos e íconos más representativos y más utilizados a nivel internacional por los regímenes comunistas: la hoz, el martillo y la estrella roja. La hoz se refiere al campesinado y la agricultura, el martillo a los obreros y la industria y la estrella roja a los diferentes grupos sociales que coexisten en una sociedad socialista: los campesinos, los obreros, el ejército, los intelectuales y la juventud.

La primera parte de la serie lleva por título: "La Hoz. Concierto para un campo de lúpulo" y se lleva a cabo en la estructura abandonada de un campo de lúpulo. El lúpulo es unos de los ingredientes principales para la fabricación de cerveza, un producto que en Chequia y en Eslovaquia goza del reconocimiento internacional de ser unos de los mejores a nivel mundial, por eso los lúpulos nobles como el Saaz y el Sladek son originarios de ambos territorios y siguen cultivándose allí. Durante la colectivización comunista la tierra fértil se acabó organizando de forma común en cooperativas agrícolas (en eslovaco: Jednotné Rol'nícke družstvo) ${ }^{4}$ y el Estado durante todo su período en el poder desde 1948 hasta 1990 dotó de subsidios a estas cooperativas con el fin de que los campesinos tuviesen un nivel de vida equiparable al de los trabajadores de las fábricas en las ciudades. Con la caída del comunismo estos subsidios se paralizan y las cooperativas caen. Eslovaquia que era la principal fuerza agrícola del país ha de reinventarse en un terreno poscomunista y es incapaz de poder competir con la producción extranjera por su superioridad técnica. En este contexto se enmarca el campo de lúpulo en el que tuvo lugar la instalación, abandonado desde el 1990 hasta la intervención en 2014, en los alrededores de la ciudad de Trenčín.

El lúpulo es una planta enredadera que crece alrededor de unos finos cables metálicos desde el suelo de manera vertical hasta una gran altura, por lo tanto el campo esta construido como una gran estructura que soporta innumerables cables metálicos que cuelgan hasta el suelo. Haciendo uso del micrófono de contacto se colocaron varios de estos piezoeléctricos en un punto previamente testado y escogido por tener las mejores características para un registro del sonido de la totalidad de la estructura, es decir, de todo el entramado metálico que conforma la estructura. Estos micrófonos se colocaron por línea a un monitor-mezclador de varios canales para registrar y amplificar. En este punto donde se recogió la vibración y por tanto el sonido de la estructura se manipularon estos varios cables metálicos, tensando y agrupándolos en un vértice. En este vértice se recogió y amplificó todo el material sonoro. De esta manera se creó una instalación de cuerdas largas creando un dibujo en el espacio con material de la estructura intervenida y haciéndose sonar a sí misma.

Por una parte, la estructura suena per se por la acción natural de los elementos del contexto y de manera especial del viento. Estaríamos hablando de un gran harpa eólica, como los trabajos de Alan Lamb que de igual manera toman registros, solo desde el aspecto documental, de elementos de infraestructuras públicas obsoletas como grandes líneas abandonadas de cable del alumbrado ${ }^{5}$. Por otra parte y a su vez, se manipula con la acción de las manos la instalación de cuerdas largas de modo que se convierte en un gran instrumento o dispositivo cordófono, que se maniobra de forma frotada, con resina de colofonia, y de forma pulsada.

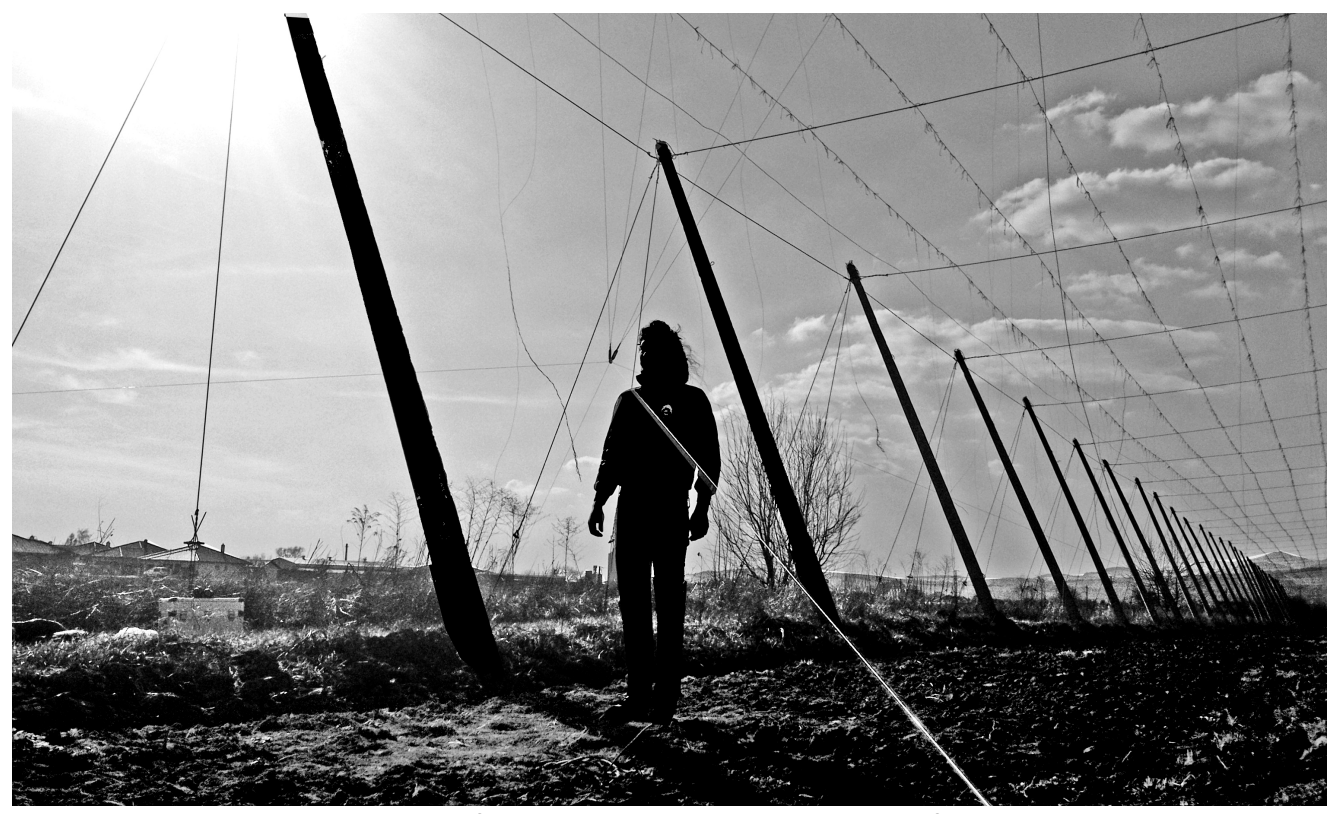

Santiago López. Concierto para un campo de lúpulo.

\footnotetext{
${ }^{4}$ TABORSKY, E. Communism in Czechoslovakia,1948-1960. 1st. ed. Princeton University Press, 1961. 402 p. L.C. Card: 61-7425.

${ }^{5}$ LAMB, A. Journeys on the winds of time [CD-audio]. USA: New Albion, 1990.
} 
La segunda parte de la serie lleva por título "El Martillo. Concierto para guitarra y un cable largo". Este concierto tuvo lugar en una antigua fábrica de alambre. La industria armamentística era la única industria pesada que existía en el territorio eslovaco y era la base económica del estado federal junto con la agricultura. En este caso, el espacio donde se llevó a cabo la intervención, fue una fábrica estatal que aprovechaba todo el material sobrante de la metalúrgica armamentística para el procesamiento del acero y todo tipo de metales y aleaciones con el fin de fabricar alambre para fines diversos. Uno de estos fines, además de los más comunes como tejido o cable eléctrico, podría haber sido con mucha probabilidad bobina de cobre, material que se utilizaba para las pastillas de las guitarras eléctricas checas que desde finales de los años 50 se consolidaban como las mejores guitarras construidas en Europa ${ }^{6}$. En el el contexto de este espacio en particular se realizó una intervención dentro la fábrica en la que se hizo uso de un largo cable o alambre metálico que ocupaba todo el espacio de forma longitudinal que se amplificó con las pastillas de una de las célebres guitarras construidas en Checoslovaquia. Se hizo uso del cuerpo completo del instrumento, una Jolana Galaxis del año 1981 construida en la fábrica estatal de Krnov y que fue conectada a un amplificador de guitarra. Con unas condiciones óptimas espaciales para una acústica con un reverbeo constante y envolvente, el sonido de la cuerda larga era frotado por una persona mientras que en el cuerpo de la guitarra otra persona manipula la cuerda de forma pulsada.

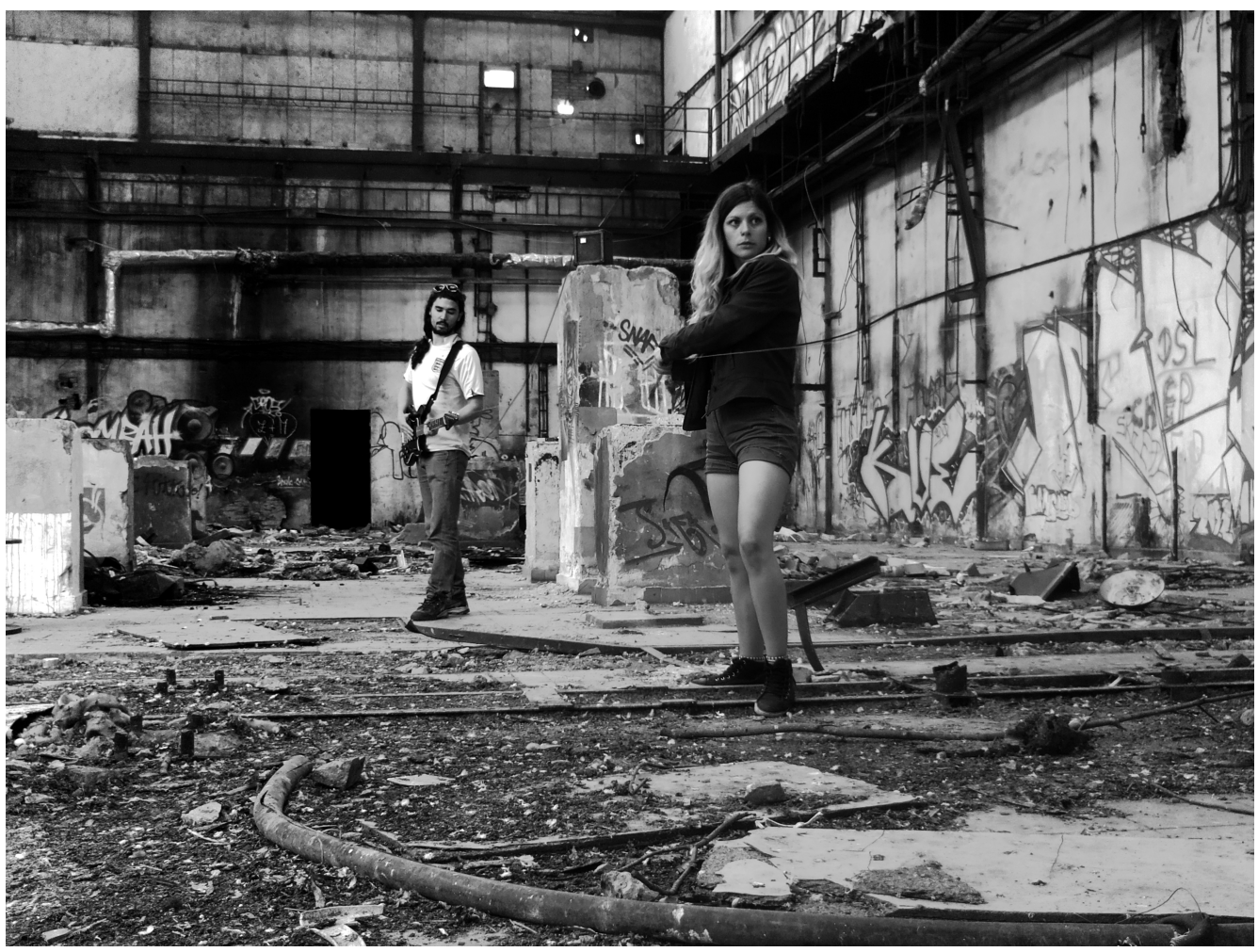

Santiago López. Concierto para guitarra y un cable largo.

La tercera parte de la serie lleva por título "La Estrella Roja. Concierto para tendederos sociales". Son una serie de micro-conciertos registrados en directo en el distrito de Dlhé Hony en Trenčín en los que que se utilizan los tendederos de largas cuerdas como instrumentos o dispositivos sonoros. Este barrio residencial, un microdistrito socialista (en checo llamado sídliště) es el primero que se construye a mediados de los años 50 como anexo urbanístico después de la Segunda Guerra Mundial en el municipio. Se caracteriza por estar formado de uniformes bloques de hormigón llamados popularmente como panelaki. Dentro de este entramado urbanístico se utilizaron los tendederos que fueron emplazados en las plazas públicas para los vecinos. Hoy, son pocas las personas que todavía les hacen uso y son pocas las piezas que todavía conservan las cuerdas y pocas las estructuras con funciones útiles para el vecindario que aun existen, como por ejemplo los armazones para sacudir las alfombras o los espacios para arreglar el coche, de esta manera se erigen actualmente en el espacio público como desechos obsoletos.

Se instalaron micrófonos piezoeléctricos en cada uno de los tendederos metálicos, estos son unos objetos con una estuctura metálica muy básica en forma de " $\mathrm{T}$ " en los que se disponen las largas cuerdas para secar la ropa y que delimitan el espacio de las plazas

\footnotetext{
${ }^{6}$ PITT, E. 2005. Guitars of the Cold War. Four Decades of Guitars from Czechoslovakia. 20 Nov. 2005. [Consulta 15 Febrero 2017]. Disponible en: http://www.junkguitars.com/stories/czech.html
} 
públicas. Despés de diseñar un itinerario, en cada una de las plazas del distrito se llevaron a cabo fugaces conciertos improvisados con un pequeño monitor.

Las tres piezas de la serie tienen un caracter site-specific a diferentes niveles, tanto físico como discursivo. Si mirasemos de forma exenta a la serie la primera pieza, en el campo de lúpulo, podríamos ubicarla en el terreno que James Meyer calificaría como sitio literal en contraposición con lo que el autor denomina como sitio funcional ${ }^{7}$. El sitio literal correspondería a la relación de la obra con el espacio físico ocupado, y la interrelación con las características estructurales del lugar y el sitio funcional haría referencia a todo lo referente al contexto, es decir, que la significación y la intención de la obera tenga que ver con lo alegórico, la memoria o lo histórico.

El concierto para un campo de lúpulo es un ejemplo claro de la explotación de los recursos del sitio, atendiendo solo a su ficisidad y sus particularidades estructurales, su sonido interno. También es un pequeño homenaje y una referencia directa a un acontecimiento importante en la historia del arte checoslovaco y a los movimientos de vanguardia de los años setenta, donde los artistas empezaban a investigar el land art y las nuevas tendencias hacia el arte procesual durante la dictadura. Nos referimos al simposio llamado Chmelnice, (campo de lúpulo), donde varios de los artistas checoslovacos más importantes del land art de la época realizaron varias intervenciones en el espacio de un campo de lúpulo ${ }^{8}$.

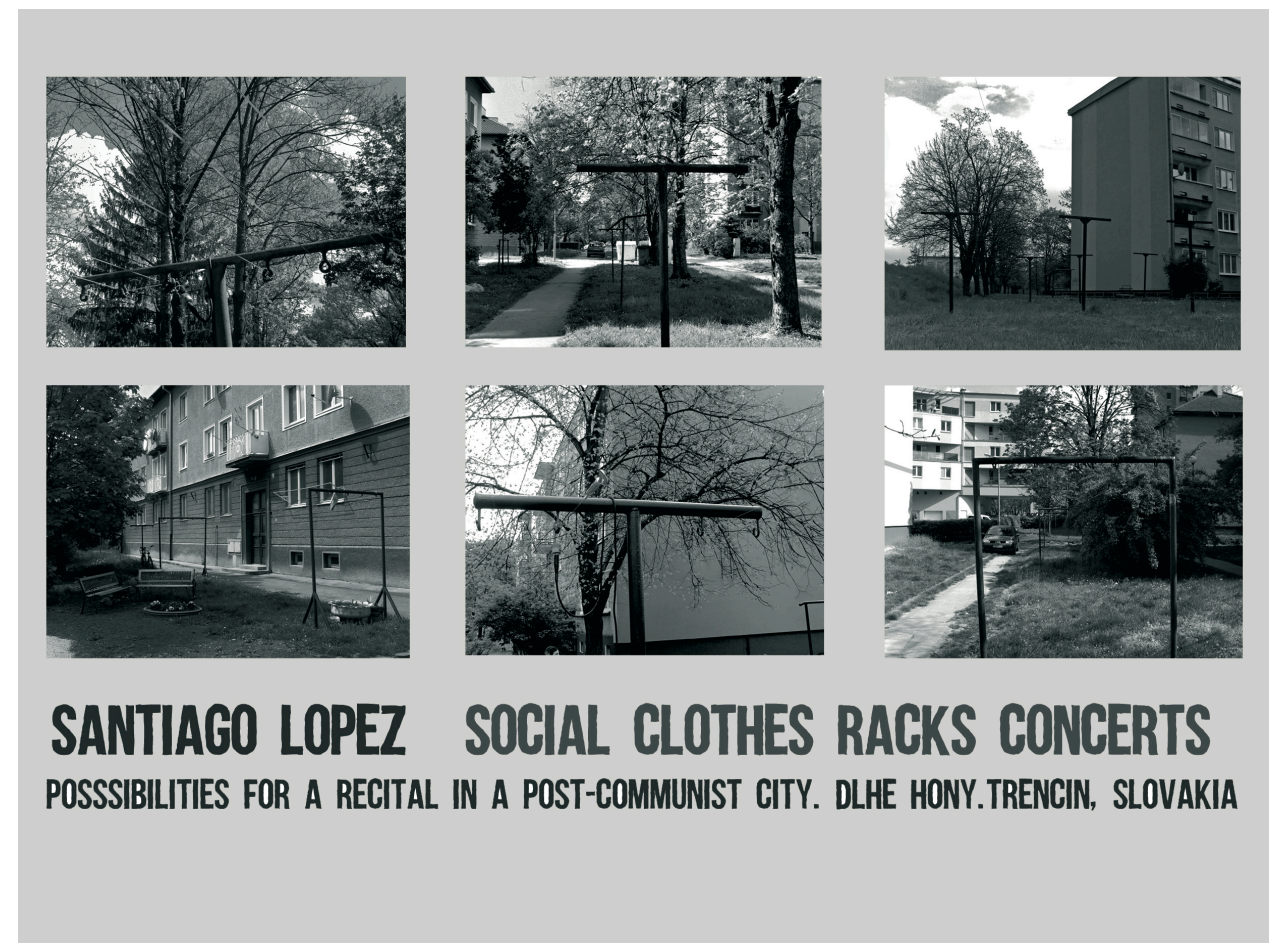

Santiago López. Espacios y estructuras para los conciertos de tendederos.

En las dos piezas siguientes de la serie podríamos hablar de unas obras sobre el lugar de un carácter más discursivo que físico. En el concierto de la fábrica existe un componente de intencionalidad importante que se refiere a la memoria y a la historia del lugar, se utiliza el objeto "alambre" la cuerda larga como un elemento que revierte la latencia del lugar volviendo a estar físicamente presente, y de igual manera ocurre con el objeto "guitarra". Existiría un tercer nivel en la pieza de los tendederos. El primer caso es físico. El segundo es físico y además discursivo porque hace referencia a la memoria del lugar, el tercero es físico, discursivo y hemos de sumarle también un elemento extra importante, lo social o relacional, lo que Miwon Kwon demanda para las obras de arte sitespecific y que las declara como "desapegadas" ${ }^{9}$, es decir que los proyectos, intervenciones o acciones sobre el lugar han de tener un componente que genere una toma de conciencia y por lo tanto intentar cambiar su realidad. En el itinerario de micro-conciertos en el distrito de Dlhé Hony, que se realizaron un domingo sin aviso previo, se utilizaron tendederos abandonados que no habían sido usados

\footnotetext{
${ }^{7}$ MEYER, J. 2000. The functional site. En: E. SUDERBURG (eds.). Space, site, intervention. Situating installation art. Minneapolis: University of Minnesota Press, pp 20-29. ISBN 0-8166-3159-X.

${ }^{8}$ MORGANOVA, P. 2015. Czech Action Art. Happenings, Actions, Events, Land Art, Body Art and Performance Art Behind the Iron Curtain. 1st. ed. Prague: Karolinum Press. ISBN 978-80-246-2317-7.

${ }^{9}$ KWON, M. 2002. One Place After Another. Site Specific Art and Locational Identity. 1st. ed. Cambridge, London: The Massachussets Institute of Technology Press. ISBN 0-262-11265-5.
} 
desde la época comunista. Durante la dictadura la libertad de reunión estaba prohibida, pero podía existir un resquicio de reunión espontánea con el vecino a la hora de tender la ropa en la plaza del barrio y por tanto este acto cotidiano se podía convertir en un espacio de convivencia social. Se puede sentir que la sociedad eslovaca y checa actual ha revertido el carácter, de la conciencia de ser una masa social para tornarse de algun modo más individualista, y por eso solo los mayores tienden la ropa en la calle, ya que en los noventa se podían instalar tendederos en las ventanas de cada particular o adquirir los tendederos de pie que pueden estar dentro de las casas. Este fue el mensaje y la lectura implícita de esta pieza para aquellos vecinos-espectadores que salieron de sus ventanas y casas para escuchar los conciertos, preguntar, conversar y que hicieron de la pieza un evento relacional.

Los espacios urbanos o industriales abandonados son, desde la visión de Smithson, lugares cargados de entropía ${ }^{10}$. Smithson establece una analogía entre las ciencias naturales y su visión de la estética cuando interpreta el concepto de entropía en sus intervenciones en espacios abandonados. La entropía sería el proceso de desintegración de las estructuras y los lugares donde se realizan las intervenciones, y la obra de arte sería el resultado de de la paralización momentánea de la entropía. Estas acciones se convierten en reciclajes del lugar, del paisaje a través de una idea expandida de monumento y por eso Smithson, al igual que en esta serie, descontextualiza estos espacios abandonados dotándoles de un sentido de un futuro a la inversa. Por ello la cita de Nabokov "el futuro es lo obsoleto al revés" que aparece en el texto de su obra "Monuments of Passaic" en la cual se registran fotografías de paisajes postindustriales ${ }^{11}$.

\section{Conclusiones.}

Si los reciclajes de Smithson se enmarcan sobre las ruinas de la sociedad del desecho capitalista, este trabajo lo haría en la entrada en vigor de la sociedad del consumo y el cambio de era. Como en el trabajo de Smithson, aquí existe una intención de reciclaje, ya no desde una perspectiva puramente visual y conceptual como lo hace Smithson, si no que hemos de añadirle el contenido de lo sonoro como material escultórico y la ficisidad del cuerpo en el registro audiovisual. Es decir, añadimos dos variables de carácter físico a este tipo de prácticas, lo sonoro y la presencia del cuerpo. Este trabajo de producción artística como investigación, postula que las prácticas del arte sonoro pueden enmarcarse dentro del site specific y al arte procesual desde la perspectiva conceptual de Smithson sobre el lugar y la entropía y en concreto, dentro de la investigación en las artes sónicas, en el uso del micrófono piezoeléctrico sobre objetos idiofónicos (los tenderos) e instalaciones de largas cuerdas metálicas.

El reciclaje de la memoria y la historia de una comunidad sería uno de los objetivos de este trabajo en el contexto de la sociedad eslovaca, que ha sufrido el cambio de ser un país completamente aislado del mundo occidental, donde el régimen esgrimía la cultura tradicional, el folk y el nacionalismo, a convertirse en terreno globalizado con sus luces y sus sombras. El símbolo del campo de lúpulo como espacio para la libertad en la dictadura, la guitarra Jolana, empresa que ya perdió la fábrica original y que construyó guitarras "pirata", es decir, guitarras que eran copias o rediseños de las americanas que ocupaban el mercado internacional, las fábricas de producto local que no han soportado el cambio, y casi lo más importante, tal como se intenta recuperar en los conciertos de tendederos en el espacio público, la vida social, las relaciones sociales que se enfriaron durante esos 41 años de dictadura, que hoy, a causa de ese carácter de reversión hacia el individualismo y de manera paradójica dentro de la sociedad global, se ha de recuperar.

\section{FUENTES REFERENCIALES.}

FRASSINETI, C. La globalización vista desde los últimos. Translated by AO García; edited by Sal Terrae. 1st. ed. Cittadela Editrice, 2000. 20 p. ISBN 84-293-1412-1.

GOTT, R. Cuba. A new story. Yale University Press, 2004. 365 p. ISBN 978-84-460-2432-3.

GRANCAY, M and GRANCAY, N. Foreign Direct Investment in Central and Eastern Europe: Post-crisis. 1st. ed; Budapest: Studies in economic transition 2017. Section 4, Szent-Iványi, pp 77-99.

KWON, M. 2002. One Place After Another. Site Specific Art and Locational Identity. 1st. ed. Cambridge, London: The Massachussets Institute of Technology Press. ISBN 0-262-11265-5.

LAMB, A. Journeys on the winds of time [CD-audio]. USA: New Albion, 1990.

\footnotetext{
${ }^{10}$ SMITHSON, R. 1996. Robert Smithson: The Collected Writings. 2nd. ed. Berkeley, Los Angeles, London: University of California Press. ISBN 0-52020385-2.

${ }^{11}$ Ibid.
} 
Posibilidades para un recital en una ciudad poscomunista. Entropología sonora en el "Tigre de los Tatra"

III CONGRESO INTERNACIONAL DE INVESTIGACIÓN EN ARTES VISUALES :: ANIAV 2017 :: GLOCAL [codificar, mediar, transformar, vivir] http://dx.doi.org/10.4995/ANIAV.2017.4567

MEYER, J. 2000. The functional site. En: E. SUDERBURG (eds.). Space, site, intervention. Situating installation art. Minneapolis: University of Minnesota Press, pp 20-29. ISBN 0-8166-3159-X.

MORGANOVA, P. 2015. Czech Action Art. Happenings, Actions, Events, Land Art, Body Art and Performance Art Behind the Iron Curtain. 1st. ed. Prague: Karolinum Press. ISBN 978-80-246-2317-7.

PITT, E. 2005. Guitars of the Cold War. Four Decades of Guitars from Czechoslovakia. 20 Nov. 2005. [Consulta 15 Febrero 2017]. Disponible en: http://www.junkguitars.com/stories/czech.html

SMITHSON, R. 1996. Robert Smithson: The Collected Writings. 2nd. ed. Berkeley, Los Angeles, London: University of California Press. ISBN 0-520-20385-2.

TABORSKY, E. Communism in Czechoslovakia,1948-1960. 1st. ed. Princeton University Press, 1961. 402 p. L.C. Card: $61-7425$. 